Instituto Internacional de Investigación y Desarrollo Tecnológico Educativo INDTEC, C.A.

DOI: https://doi.org/10.29394/scientific.issn.2542-2987.2017.2.5.17.321-339

OAI-PMH: http://www.indteca.com/ojs/index.php/Revista Scientific/oai

\title{
Reutilizando el Material Reciclaje como Estrategia Didáctica para Fortalecer los Valores Ambientales
}

Autor: Yudit Zaida del Carmen Alarcón de Palma Universidad Pedagógica Experimental Libertador, UPEL yuditalarcon15@gmail.com

Barinas, Venezuela

\section{Resumen}

El artículo tuvo como centro de interés implementar la reutilización del material reciclaje como estrategia didáctica para fortalecer los valores ambientales a los estudiantes de la Escuela Básica Nacional "Adolfo Moreno" de la parroquia Barinitas, municipio Bolívar, estado Barinas. Año Escolar 2014 - 2015. El estudio se sustentó bajo el paradigma de investigación cualitativa y en el tipo investigación acción. Desde este punto de vista, el estudio se enfoca en esta modalidad acción participativa, se circunscribe en los llamados diseños de campo. El estudio por sus características se cumplió en las siguientes fases: Diagnóstico, planificación, ejecución, evaluación y sistematización. Por último, se puede señalar que con la aplicación de estrategias didácticas en la reutilización de material reciclaje para el fortalecimiento de los valores ambientales a los estudiantes de la Escuela Básica Nacional "Adolfo Moreno"; se estarán incorporando a los padres y representantes además de diversos actores educativos para aplicar las actividades involucradas en la propuesta la cual busca un cambio de actitud para mejorar a través de acciones prácticas del manejo de normas y practicas conservacionistas, para lograr un cambio ambientalista en la institución mediante técnicas, procedimientos teóricos y prácticos para afianzar los conocimientos en beneficio de manejar adecuadamente los materiales reciclables.

Palabras clave: reutilizando; material reciclaje; estrategia didáctica; fortalecer los valores ambientales. 


\title{
Reusing Recycling Material as Teaching Strategy to Strengthen Environmental Values
}

\begin{abstract}
The study was centered interest implement recycling reuse the material as a teaching strategy to strengthen environmental students "Adolfo Moreno" National Basic School Barinitas parish, municipality Bolivar, Barinas state values. School Year 2014 - 2015. The study was based on the paradigm of qualitative research and research in action type. From this point of view, the study focuses on participatory action this mode, it is limited in so-called field layouts. The study its characteristics was fulfilled in the following phases: diagnosis, planning, implementation, evaluation and systematization. Finally, it can be noted that the implementation of teaching strategies reuse recycle material for strengthening environmental students "Adolfo Moreno" National Basic School values; They will be incorporating parents and guardians as well as various educational actors to implement the activities involved in the proposal which seeks to change attitudes to improve through practical actions management standards and conservation practices to achieve an environmental change in institution through technical, theoretical and practical knowledge to strengthen the benefit of recyclables properly handle procedures.
\end{abstract}

Keywords: reusing; recycling materials; teaching strategy; strengthen environmental values.

Date Received: 17-02-2017

Date Acceptance: 18-04-2017 


\section{Introducción}

A nivel mundial la contaminación ambiental se ha convertido en unos de los más grandes problemas que existen actualmente en el planeta introduciendo como consecuencia la contaminación de agua, aire, y provocando enfermedades a las comunidades; así como a la población en general. Por tal razón, en distintos países del mundo han tomado la iniciativa de crear planes de acciones contra la contaminación del ambiente por medio del reciclaje dando el ejemplo para que las demás naciones tomen conciencia y contribuyan a la disminución de la problemática.

Cabe destacar, que la Organización de las Naciones Unidas (1997), en la ciudad de Kioto - Japón, realizó una conferencia con el fin de "Acordar medidas y compromisos para la reducción de la contaminación ambiental" (p/s.n). En esta reunión más de 160 países acordaron disminuir la cantidad de dióxido de carbono hasta el año 2012, lo que se consideró de trascendencia para los países; así como una búsqueda de un cambio para el proceso socioambiental.

Con estos compromisos se desea mejorar o disminuir los índices de contaminación ambiental de forma gradual, mediante planes de gestión de contaminantes y el control de desechos emanados por la industria hacia la atmósfera. También se espera reducir la gestión de residuos sólidos urbanos, aprovechando los materiales reciclables, es decir implementar estrategias didácticas que conlleven a valorar ambiente.

Por lo tanto, la educación ambiental debería jugar un papel fundamental desde el inicio de la educación formal, para así relacionar hombre, su forma de vida y ambiente, buscar un cambio de actitud; así como establecer un cambio de conciencia, sensibilización enfocado en la proyección de un ciudadano con fines conservacionista, sobre la importancia de resguardad para el futuro, con el firme compromiso de mejorar la calidad de vida. En tal sentido, Dohogne (2010), plantea que: 
La educación ambiental debe ser: en los primeros años de nuestras vidas, ya que son muy importante al momento de desarrollar los hábitos y el conocimiento. Un conocimiento que si viene de la mano de estrategias divertidas se fija en nuestra memoria con mayor facilidad. (pág. 16).

Es importante sensibilizar a la población, iniciando desde los primeros años escolares, implementando estrategias y talleres para reconocer los problemas y buscar soluciones con el propósito de promover actitudes y valores ambientales, para ser buenos ciudadanos. El impacto de los residuos en el ambiente es la inmensa cantidad que el hombre día tras día a acumulado lo que implica que muchos de estos materiales sólidos no son biodegradables, por eso la permanencia de los mismo en la naturaleza que no pueden ser atacados por microorganismos. Esta característica hace que ocupen espacios en los botaderos por tiempos indeterminados, generando diferentes tipos de dificultades. Según Martínez (2010), asevera:

La acumulación de residuos sólidos conlleva a la búsqueda de más espacios para la acumulación de basura utilizando zonas que pudiesen tener otro uso, la producción energética y la maquinaria empleada para la fabricación de todos los residuos sólidos desechados, es otro factor que se debe cuestionar el ser humano, como principal responsable del desequilibrio ambiental (pág. 68).

Apoyando lo expresado por el autor, vale comenta que lo ideal para no agredir al ambiente es destinar cada residuo a su tratamiento, proceso por el cual se puede salvar grandes cantidades de recursos naturales no renovable que deban tener una segunda transformación y utilidad. Asimismo, en el Estado Barinas el problema de residuos sólidos está latente día a día lo que ocasiona numerosos daños ambientales, debido al crecimiento de la población y la falta de sensibilización y el desconocimiento de la utilización del reciclaje. Al respecto Fuente (2006), señala en su trabajo de investigación que: 
Debido a los altos volúmenes de residuos sólidos producidos en las grandes ciudades y a la deficiencia en los procesos de recolección, clasificación y disposición final, la mayoría de los rellenos sanitarios representan un problema en vez de una solución, y algunos de ellos han colapsado. (pág. 15).

Una de las consecuencias que ha generado la cantidad de desechos sólidos por las comunidades, ha sido el deterioro ambiental y por ende la salud de la población, ocasionando enfermedades en el ser humano y deteriorando elementos fundamentales del medio ambiente tales como: agua, aire, suelo, flora y fauna que constituyen el patrimonio ambiental de la región; convirtiéndose en una amenaza significativa para el estado para encaminarse al desarrollo sustentable.

Por lo ante señalado, se puede decir que es necesario buscar medidas como incentivo para concientizar y dar a conocer la reutilización de material reciclaje como estrategia didáctica para fortalecer los valores ambientales a los estudiantes de la Escuela Básica Nacional Adolfo Moreno, con el propósito de conservar el ambiente y prevenir la contaminación del mismo; puesto que se encuentra una serie de causas que van desde la escasa o deficiente manera en que se ha promovido la reutilización; así como una cultura para la selección de los residuos sólido.

Por esta razón, esto se orienta en efectuar un trabajo organizado a través de la implementación de estrategias para mantener limpio las áreas verdes, las aulas y todos aquellos espacios físicos; produciendo embellecimiento de la escuela y en la comunidad, con el propósito de contribuir para un ambiente sano y agradable; el cual requieren quienes habitan en lugares que cada día se encuentran con cantidades de desperdicios y se convierten en un problema de sanidad urbana.

Por todo lo anteriormente expuesto, sumado a mejorar los niveles de contaminación ambiental presentados y producidos por los desechos sólidos, 
se plantea las siguientes interrogantes: ¿Cuáles son los conocimientos de la reutilización de material reciclaje como estrategia didáctica para fortalecer los valores ambientales que tienen los estudiantes de la Escuela Básica Nacional Adolfo Moreno"?, ¿Quiénes participaran en la planificación del impacto del reutilizar material reciclaje para fortalecer los valores ambientales a en los estudiantes de la Escuela Básica Nacional "Adolfo Moreno"?.

De igual manera, se desatan ¿Qué estrategias didácticas se pueden aplicar con la reutilización de material reciclaje para fortalecer los valores ambientales a los estudiantes de la Escuela Básica Nacional “Adolfo Moreno”, ¿Cómo evaluar las actividades sistemática que debe realizarse en cada una de las tareas planificadas con la reutilización de material reciclaje como estrategia didáctica para fortalecer los valores ambientales a los estudiantes de la Escuela Básica Nacional "Adolfo Moreno?, ¿Cómo lograr que las estrategias didácticas de reutilización de material para reciclaje puedan fortalecer los valores ambientales a los estudiantes de la Escuela Básica Nacional "Adolfo Moreno" de la Parroquia Barinitas?.

En relación con el estudio se encuentra los objetivos de la investigación, destacándose el objetivo general como fue: Implementar la reutilización del material reciclaje como estrategia didáctica para fortalecer los valores ambientales a los estudiantes de la Escuela Básica Nacional "Adolfo Moreno" de la parroquia Barinitas, municipio Bolívar, estado Barinas. Año Escolar 2014 -2015 .

\section{Contexto Teórico}

En el desarrollo del antecedente del problema en investigación se consideran algunos estudios que enfocan aspectos relacionados con el tema. El desarrollo permitió dar una visión amplia de las técnicas, métodos y conclusiones encontradas en otras investigaciones, referentes al manejo y 
reutilización de los desechos sólidos, y los valores ambientales que aportan un análisis importante para el desarrollo de este estudio.

Por su parte, Bravo (2012), en el trabajo de grado "La Política y Gestión Municipal de Desechos Sólidos en el municipio Rosario de Perijá del estado Zulia". El objetivo del estudio fue analizar la política y gestión municipal de desechos sólidos en el municipio Rosario de Perijá del estado Zulia, con la finalidad de contribuir significativamente a la preservación del medio ambiente y a la disminución de enfermedades que afectan directamente a la comunidad.

Se concluye, que el inadecuado manejo de los desechos sólidos por parte del ente municipal, causan graves efectos de contaminación ambiental, que afectan la calidad de vida y la salud de los habitantes de la población Perijanera y los pueblos circunvecinos; generando una serie de enfermedades, especialmente en la población infantil. Se recomienda generar políticas municipales que estimulen y fomenten sistemas de gestión para la reutilización productiva de los desechos sólidos con miras al reciclaje con la participación de la comunidad.

Cabe destacar, en el estudio presente que es importante consolidar la concientización a la comunidad ciudadana para el buen manejo de reutilización de los desechos sólidos con el propósito de eliminar la contaminación ambiental y evitar las enfermedades a los habitantes de esta manera de cambio pueden apreciar un ambiente limpio y agradable; aceptable a las condiciones del hombre y el ambiente.

\subsection{El Reciclaje}

En la actualidad y gracias a las nuevas tecnologías, el reciclaje es una de las alternativas utilizadas por el hombre en la reducción del volumen de desperdicios sólidos. Este proceso consiste en volver a utilizar materiales que fueron desechados, y que aún son aptos para elaborar otros productos o refabricar los mismos. Este hecho implica el regreso de materiales 
recuperados, que no se pueden usar más en el proceso manufacturero en sus etapas primarias, como la molienda y la fundición; pero si para diversas actividades que contribuyan a disminuir el deterioro ambiental y optimización de la calidad de vida. De acuerdo a Huilca, (2015), el reciclaje es:

Un conjunto de acciones que realiza la naturaleza y el hombre sobre diferentes materiales para volver a recuperarlos y utilizarlos. En la naturaleza, gracias a estos procesos de reciclaje, los nutrientes esenciales para la vida, vuelven a circular en los diferentes ecosistemas de la Tierra, ya sean estos terrestres, acuáticos o aéreos (pág. 33).

De acuerdo al aporte del autor en la definición del reciclaje cabe destacar el origen, proceso y como el hombre puede utilizarlo en cuanto a la materia orgánica (las plantas), así mimo menciona los materiales fabricados por el hombre, ya que es parte de los seres humanos en cuanto a la colaboración que le podemos brindar desde los hogares separando la basura en orgánica e inorgánica.

Los nutrientes se mueven en estos distintos ambientes pasando por los organismos para regresar nuevamente al ambiente. Un ejemplo es la materia orgánica de los seres vivos que al morir vuelve a ser utilizada por las plantas, al ser degradada por los microorganismos en compuestos minerales simples que incorporan las plantas para formar sus estructuras y realizar sus funciones. Cabe destacar que, según Albarracin (2010), explica "los materiales reciclables son los metales, vidrio, plástico, papel y cartón entre otros" (pág. 12). Si se quiere colaborar en el reciclaje de materiales, es necesario comenzar en los hogares separando la basura en orgánica e inorgánica. No desechar los cuadernos escolares si aún tienen hojas utilizables, reutilizar las botellas de agua y otros frascos y recipientes. Juntar las latas de refresco para venderlas a algún sitio recolector de aluminio o adornar algunas latas para regalarlas como lapiceros, es decir, que se utilicen con ingenio para comenzar a reciclar. 


\subsection{Significado de las $3 R$}

Al plantarse elementos del trabajo ambiental se encuentra las tres erres (3R) es una norma para cuidar las condiciones ambientales, específicamente con el propósito para reducir el volumen de residuos o basura generada. En este caso, Aspurua (2010), explica que "Las 3R te ayudan a desechar menos basura, ahorrar dinero y ser un consumidor más responsable, así reduciendo tu huella de carbono" (pág. 27).

Reducir: radica en efectuar cambios en la conducta cotidiana para generar una menor cantidad de residuos, por ejemplo, preferir la compra de productos de buena calidad y durables; comprar sólo lo que realmente se necesita; llevar bolsas de género cuando se va de compras, evitar productos con envoltorios excesivos. Una manera importante de reducir los residuos es la recuperación de la materia orgánica para compost. Reducir también significa rechazar productos cuyo uso o cuya disposición final resultan contaminantes, como pilas o detergentes optando siempre que se pueda por soluciones alternativas (artefactos conectables a la corriente eléctrica, productos de limpieza natural o de bajo impacto contaminante).

Reutilizar: consiste en dar el máximo de usos a un producto antes de considerarlo basura. Se puede reutilizar un producto para la misma función que fue concebido. Por ejemplo: las botellas de bebida retornables. También es posible reutilizar un producto para una función diferente, por ejemplo, una botella de bebida puede ser reutilizada como macetero.

Reciclar: consiste en devolver al ciclo productivo los residuos que pueden ser reutilizados como materia prima, por ejemplo: papeles, cartones, vidrios, materiales plásticos. El proceso de reciclar ahorra recursos naturales y energía.

En virtud a lo expuesto, se tiene como factores de interés en lo inherente a cuestiones fundamentales en el reciclaje se incluyen la identificación de los materiales que se van a desviar del flujo de residuos, las posibilidades de 
reutilización de materiales reciclable específicamente de los compradores de materiales recuperados.

\subsection{Identificación de las Posibilidades de Reutilización y Reciclaje}

En relación con el reciclaje, se considera que estos pueden ser en la mayoría de los casos, elementos recuperados, en algunos casos de menor calidad a los materiales vírgenes, por lo que el precio en el mercado tiene que ser atractivo para los compradores. También se crean mercados con una legislación que desarrolle una demanda a largo plazo y con los avances tecnológicos.

De acuerdo a Albarracín (2010), explica: "Los gestores encargados del desarrollo de un programa de reciclaje deben tener en cuenta los mercados para los materiales recuperados, la infraestructura de recogida y el coste global" (pág. 12). Los mercados para los materiales recuperados existen solamente cuando los fabricantes o procesadores necesitan estos materiales o pueden usarlos como sustitutos rentables de materias primas; por tanto, el mercado depende de la calidad de los materiales, de la capacidad global de la industria y del coste de las materias primas en competencia.

En tal sentido, se considera a los recicladores como un soportar el valor del transporte a estas instalaciones centralizadas. Es por eso es la recogida y la vía hasta los compradores, comparado con el precio pagado por los materiales recuperados, es normalmente la razón de que las comunidades más pequeñas no hayan sido capaces de mantener programas de reciclaje sin subvenciones.

\subsection{Influencia en Venezuela del Reciclaje}

El Reciclaje en Venezuela señalado por Barreto (1990) es una forma de Subsidio Conservacionista a los Clubes del Ministerio de Agricultura, los programas de educación y de protección ambiental del Ministerio de Ambiente 
y el programa hacia una sociedad ecológica que funciona desde 1980 en el Centro de reciclaje de Caricuao. El Sistema de Reciclaje del Estado Carabobo (SIDEC), el Instituto de Saneamiento Ambiental del Municipio Sucre del Distrito Capital y algunos destacamentos del Ejército en las zonas fronterizas; de muchas de estas iniciativas se ha perdido el rastro y algunas aún sobreviven con sus altibajos.

Ante esto, algunas comunidades, como el Paují y Cantarrana en el estado Bolívar y La Azulita en Mérida; movimientos vecinales, en Caurimare, el Cafetal y Manzanares en Caracas y empresas con negocios asociados a la recuperación de materiales como Cotécnica, Fospuca, Owens Illinois, Kimberly-Clark Venezuela, Fundación Papyrus de Manufacturas de Papel C.A. (MANPA), Productos de Vidrio S.A. (PRODUVISA) y Ecoplast- adelantan experiencias en este sentido.

\subsection{Valores Ambientales}

Lo valores ambientales, se asocia en un estatus dirigido a la formación de individuos para la socialización, tomándose como elemento de importancia la soberanía e unificación al desarrollo, pero desde su conciencia crítica. Según Rosales y García (2011), definen a los valores ambientales como "Un conjunto de actividades que involucran el cuidado y mantenimiento de los aspectos que forman parte del contexto humano y natural" (pág. 22).

Ante esto, la educación siempre va articulada a la idea de desarrollo relacionado y recomendable al hecho del mejoramiento de las condiciones que presenta la naturaleza. Según Payá (1997), es importante resaltar "El proceso educativo ante el aspecto axiológico es entendida como aquello que busca conducir hacia el bien o deseable, sigue un modelo ideal para alcanzar el objetivo último de cada etapa, permitiendo el desarrollo integral de la persona" (pág. 45). 
Educar en valores es promover, sin imposición y a través de la aceptación de cada individuo, conceptos que al formularlos produzcan motivaciones, intenciones, propósitos, adhesiones o rechazos, conducentes a formar ciudadanos críticos, comprometidos con ideales de justicia y respeto por los otros y por el ambiente.

Con respecto a la definición de los autores, cabe destacar la importancia de impartir la educación en valores ambientales a los estudiantes de todos los niveles con las políticas educativas integradoras a través de contenidos y estrategias donde el niño y niña aprenden a valorar el ambiente desde temprana edad para obtener como resultado una conciencia ecológica, sean críticos, reflexivos y el respeto al ambiente.

\section{Metodología}

El estudio se sustenta bajo el paradigma de investigación cualitativa y en el tipo investigación acción. Cabe señalar que la investigación cualitativa está orientada al estudio en profundidad de la compleja realidad social, por lo cual en el proceso de recolección de datos el investigador va acumulando textos provenientes de distintas técnicas, para Goetz y LeCompte (1988), el análisis de esta información debe ser abordado de forma sistemática, orientado a generar constructos y establecer relaciones entre ellos, constituyéndose esta metodología. En relación con esto, Rojas (2010), en relación a la investigación cualitativa señala:

La investigación cualitativa se orienta hacia el estudio de problemas relacionados con la experiencia humana individual y colectiva; fenómenos sobre los que se conoce poco y se aspira comprender en su contexto natural. El diseño en la investigación cualitativa tiene su carácter flexible y emergente, que implica tomar decisiones en el contexto durante el proceso. (pág. 58) 
En este sentido, la presente investigación busca que el investigador se sumerja en la realidad del contexto, permitiendo que se conviva, comparta, reflexione y se dialogue con la realidad subjetiva en sí misma, siendo el objeto de estudio.

Desde este punto de vista, el estudio se enfoca en esta modalidad, investigación acción participativa, puesto que permite desarrollar un proceso de investigación cooperativo y comprometido con la realidad social, generando acciones de tipo formativo y desarrollo de la personalidad de cada individuo sensibles a los problemas ambientales en la sociedad y que contribuyan a mejorar situaciones problemáticas en espacios educativos o comunitarios. En tal sentido, Rojas (2010), expresa que la investigación Acción-participativa se caracteriza por:

Forma parte de una situación social específica en la cual se ha detectado una problemática. Desarrolla un proceso dialectico en el cual la acción genera conocimiento y la investigación conduce a la transformación. En el proceso trabajan de forma conjunta y mancomunada los integrantes de la comunidad, los investigadores, promotores y animadores. La dinámica de participación genera un compromiso de participación genera un compromiso, tanto del investigador como del resto... (pág. 51).

La investigación se circunscribe en los llamados diseños de campo, porque a través de él se pretende ir al lugar donde ocurren los hechos en la población o ambiente de estudio, por eso según, el Manual de Tesis de Grado Universidad Nacional Experimental Rómulo Gallegos (2006), cuando dice:

Se entiende por investigación de campo al análisis sistemático de problemas en la realidad con el propósito bien sea de describirlos, interpretarlos, entender su naturaleza y factores constituyentes, explicar sus causar y efectos o predecir sus ocurrencias, los datos de interés son recogidos en forma de la realidad; en este sentido, se trata de investigación a partir de los datos originados o primarios (pág. 97). 
De acuerdo a lo acotado por el autor, la investigación de campo permite obtener datos directamente de la realidad estudiada, además de poder analizarlos y entender su naturaleza. En este caso, los datos fueron obtenidos directamente de los estudiantes y docentes de la Escuela Básica Nacional "Adolfo Moreno"; porque en ella se describe las acciones para integrarlos.

Por otra parte, se menciona que los datos fueron recabados a través de distintas técnicas e instrumentos en la propia institución donde se desarrolla la investigación, esto se logró mediante la guía de entrevista que va dirigida a ellos y su finalidad es almacenar información que puede ser revisada y evidenciada de acuerdo a la situación problemática existentes en la institución.

El estudio por sus características se cumplió en las siguientes fases, que de acuerdo a Martínez (2010): "las etapas de actividades son cinco: Diagnóstico, planificación, ejecución, evaluación y sistematización, a continuación, se describe lo realizado en cada una de ellas: Fase I. Diagnóstico, Fase II. Planificación, Fase III. Ejecución, Fase IV. Evaluación Fase y V. Sistematización.

El esquema metodológico propuesto por este autor, está constituido por las siguientes fases:

Fase I. Diagnóstico: En esta fase se identifica la situación a través de la recolección de la información con los instrumentos y técnicas seleccionadas para determinar la necesidad de reutilización del material reciclaje, para el fortalecimiento de los valores ambientales de la Escuela Básica Nacional "Adolfo Moreno".

Fase II. Planificación: se organizaron las acciones a seguir como alternativas de solución para la problemática planteada, tomando en cuenta todos los factores que pudieron influir como: el tiempo, los recursos económicos, las personas dispuestas a participar en el trabajo entre otros. En este caso específico, acciones para la reutilización del material reciclaje como 
estrategia didáctica para fortalecer los valores ambientales a los estudiantes de la Escuela Básica Nacional "Adolfo Moreno".

Fase III. Ejecución: es la fase de puesta en práctica de las acciones, del enfrentamiento con el o los problemas, es la que permite el conocimiento más profundo de la realidad, pues éste sólo se logra cuando se intenta transformarla. En este sentido, la investigadora implementa las acciones necesarias para resolver las situaciones problemáticas, actuando y recogiendo informaciones y datos, que son procesados, evaluados e interpretados. Esto es lo más importante de este método pues ayuda a las personas a identificar sus problemas, a determinar cuáles son las verdaderas causas de los mismos y a actuar en consecuencia para lograr su solución. En este particular, se realizaron las actividades enfocadas a mejorar la situación que presenta la población de estudio.

Fase IV. Evaluación: esta fase se presenta simultáneamente con la ejecución, pero en general hay simultaneidad entre las distintas etapas, pues, constantemente se están evaluado los planes y las ejecuciones; haciendo las correcciones necesarias. Sin embargo, al finalizar algunas de las fases comprendidas en el proceso de ejecución, es necesario detenerse a evaluar los resultados, analizar los éxitos y fracasos, en consecuencia, rectificar el rumbo, corregir los planes o establecer nuevas metas, con el propósito de conocer si las acciones que se ejecuten fortalecieron la reutilización del material reciclaje, para el fortalecimiento de los valores ambientales, permitiendo conocer si los pasos realizados cumplieron con el cometido para el cual fueron efectuados.

Fase V. Sistematización: En esta fase se realizó la discusión y reflexión de todas las fases desarrolladas en la investigación, con la finalidad de obtener conclusiones valiosas que permitan tener una visión global y profunda del problema, facilitando evidenciar las necesidades de involucrar a los estudiantes y docentes, con el cumplimiento a las tareas referidas al trabajo 
de campo donde el aprender haciendo transforma las debilidades en fortalezas.

\section{Consideraciones Finales}

De acuerdo a la revisión bibliográfica, los objetivos propuestos y la aplicación de los instrumentos se efectúan las siguientes conclusiones:

En la realización del diagnóstico y de los testimonios de los encuestados, se recogen las evidencias, la necesidad de reutilización del material reciclaje, para el fortalecimiento de los valores ambientales en la Escuela Básica Nacional "Adolfo Moreno" de la Parroquia Barinitas, todo ello, relacionadas con la aplicación del instrumento, y el análisis de las respuestas dadas de manera cualitativa; permitiendo recoger la información de cada jornada diaria, dentro del cual se observó deficiencia en relación a las actividades referidas a una conciencia ecológica mediante la reutilización de material de reciclaje.

En la entrevista referida a planificar estrategias didácticas en la reutilización de material reciclaje para el fortalecimiento los valores ambientales a los estudiantes de la Escuela Básica Nacional "Adolfo Moreno", los argumentos dados por los sujetos de estudio se logró constatar que, se demanda de técnicas y procedimientos teóricos y prácticos para afianzar los conocimientos en cuanto al reciclaje, su clasificación y reordenamiento, fortalecimiento los valores ambientales así aumentar el trabajo ecológico, o preservar el ambiente

En referencia a la ejecución de estrategias didácticas en la reutilización de material reciclaje para el fortalecimiento de los valores ambientales a los estudiantes de la Escuela Básica Nacional "Adolfo Moreno", ofrece una alternativa para aplicar estrategias, recursos, técnicas y medios que le permitan desarrollar acciones favorables, es decir, la optimización del trabajo diario basada en una conciencia ecologista, en beneficio de la escuela y la 
comunidad. Asimismo, los docentes refieren la introducción de diversos recursos entre foros, carteleras, afiches charlas recetarios, trípticos, asesoría técnica, para cultivar una función didáctico-pedagógica y productiva.

Seguidamente y en función de la valoración del trabajo realizado en la reutilización de material reciclaje como estrategia didáctica para el fortalecimiento los valores ambientales en los estudiantes; se destaca la importancia del ordenamiento y clasificación de los desechos reciclables en la institución: formando en los estudiantes normas y hábitos conservacionista que contribuyan con un ambiente agradable de forma permanente, dentro del contexto.

Por último, se puede señalar que con la aplicación de estrategias didácticas en la reutilización de material reciclaje para el fortalecimiento de los valores ambientales a los estudiantes de la Escuela Básica Nacional "Adolfo Moreno"; se estarán incorporando a los padres y representantes además de diversos actores educativos para aplicar las actividades involucradas en la propuesta la cual busca un cambio de actitud para mejorar a través de acciones prácticas del manejo de normas y practicas conservacionistas, para lograr un cambio ambientalista en la institución mediante técnicas y procedimientos teóricos y prácticos para afianzar los conocimiento en beneficio de manejar adecuadamente los materiales reciclables.

\section{Referencias}

Albarracín, J. (2010). Diagnóstico de la situación turística del Biocorredor Yanuncay mediante la metodología del marco lógico. Universidad de Cuenca. México.

Aspurua, T (2010). Las 3R. Tercera Edición. Editorial PANAPO. Caracas. Barreto, J. (1990). Subsidio Conservacionista a los Clubes del Ministerio de Agricultur. Centro de reciclaje de Caricuao. Caracas. 
Bravo A, L. (2012). La Política y Gestión Municipal de Desechos Sólidos en el municipio Rosario de Perijá del estado Zulia. Universidad del Zulia. Maracaibo, Venezuela.

Dohogne, E. (2010). La Educación Ambiental. Editorial Buenaventura. Tercera edición. Caracas.

Fuente, H. (2006). La Investigación y el Investigador Eficaz. Segunda Edición. UPEL. Caracas.

Goetz, J. y LeComte, M. (1988). Etnografía y diseño cualitativo de la investigación. Madrid: Morata.

Huilca, G. (2015). Elaboración y aplicación de una guía para la construcción de material didáctico con recursos del medio "manitos creadoras", para el desarrollo de la psicomotricidad en los niños/as del centro Infantil del Buen Vivir "Gotitas de Dulzura", cantón Riobamba, en el periodo lectivo 2013 - 2014. Universidad Nacional de Chimborazo.

Payá, H. (1997). Axiología de la Educación. Editorial Maya. México.

Rojas B. (2010). Investigación Cualitativa. Segunda edición Caracas. FEDUPEL. Caracas. 


\section{Yudit Zaida Del Carmen Alarcón De Palma \\ e-mail: yuditalarcon15@gmail.com}

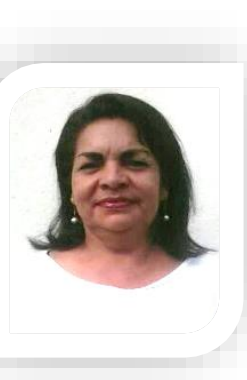

Nacida en el Estado Barinas, Venezuela. El 15 de mayo de 1971, residenciada en Barinitas, Municipio Bolívar del Estado Barinas, Venezuela, cursé estudios en la Universidad Católica Cecilio Acosta en el año 20042008, obteniendo el Titulo de Licenciada en Educación Integral. Actualmente, laboro como Subdirectora Académica en la Escuela Básica Nacional "Adolfo Moreno" ubicado en el Sector El Bucaral de Barinitas. Cursando estudios de Maestría en Educación Ambiente y Desarrollo por la Universidad Pedagógica Experimental Libertador. Instituto de Mejoramiento Profesional del Magisterio (UPEL) Núcleo Académico Barinas. 